\title{
Research Paper: Paired-pulse Inhibition and Disinhibition of the Dentate Gyrus Following Orexin Receptors Inactivation in the Basolateral Amygdala
}

\author{
Esmaeil Akbari $^{1,2}$ (D), Narges Hosseinmardi ${ }^{3,4}$ (D), Motahareh Rouhi Ardeshiri ${ }^{1,2^{*}}$ (D)
}

1. Immunogenetics Research Center, School of Medicine, Mazandaran University of Medical Sciences, Sari, Iran

2. Department of Physiology, School of Medicine, Mazandaran University of Medical Sciences, Sari, Iran.

3. Department of Physiology, School of Medicine, Shahid Beheshti University of Medical Sciences, Tehran, Iran.

4. Neurophysiology Research Center, School of Medicine, Shahid Beheshti University of Medical Sciences, Tehran, Iran.

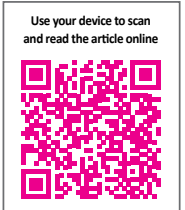

Citation Akbari, E., Hosseinmardi, N., \& Rouhi Ardeshiri M. (2021). Paired-pulse Inhibition and Disinhibition of the Dentate Gyrus Following Orexin Receptors Inactivation in the Basolateral Amygdala. Basic and Clinical Neuroscience, 12(6), 827-836. http://dx.doi.org/10.32598/bcn.12.6.1460.1

http://dx.doi.org/10.32598/bcn.12.6.1460.1

Keywords:

Basolateral amygdala, Orexin receptors, Learning, Local circuits

\section{$\underline{\text { AB S T RA C T }}$}

Introduction: The Basolateral Amygdala (BLA) substantially affects neuronal transmission and synaptic plasticity processes through the dentate gyrus. Orexin neuropeptides play different roles in the sleep/wakefulness cycle, feeding, learning, and memory. The present study aimed to investigate the function of the orexin receptors of the BLA in the hippocampal local interneuron circuits.

Methods: For this, the region's paired-pulse responses from the Dentate Gyrus (DG) were recorded. Within the procedure, SB-334867-A $(12 \mu \mathrm{g} / 0.5 \mu \mathrm{L})$ and TCS-OX2-29 $(10 \mu \mathrm{g} / 0.5 \mu \mathrm{L}$ (orexin $1 \& 2$ receptors antagonists, respectively), were administered into both sides of the BLA areas of the rat brain. Dimethyl Sulfoxide (DMSO) was used as the solvent in the control animals with a volume of $0.5 \mu \mathrm{L}$.

Results: Our data indicated that the Paired-pulse (PP) responses were not affected by the inactivation of the orexin receptors of the BLA.

Conclusion: Due to not observing any significant changes in the short form of synaptic plasticity, after inactivation of the orexin system of the BLA, we hypothesize that the orexinergic fibers to the basolateral part of the amygdala influence the long-term synaptic efficacy; however, the primary processing of information in short-term plasticity model is not affected by the same system. The elementary processing of the data by the amygdala might happen through the action of other neurotransmitter systems.

\section{* Corresponding Author:}

Motahareh Rouhi Ardeshiri, PhD.

Address: Immunogenetics Research Center, School of Medicine, Mazandaran University of Medical Sciences, Sari, Iran.

Tel: +98 (11) 33543081

E-mail:s.rouhi89@gmail.com 


\section{Highlights}

- The neuronal transmission of DG following orexin receptors antagonism of the BLA.

- Paired-pulse responses were not affected by the orexin 1 receptors antagonism.

- Paired-pulse responses were not affected by the orexin 2 receptors antagonism.

\section{Plain Language Summary}

The orexinergic system has modulatory effects by sending projection fibers to several parts of the brain, such as the hippocampus and amygdala. Orexin neuropeptides activate basolateral amygdala neural circuits during different arousal states. Although, this system plays a vital role in creating appropriate behavioral reactions, the primary processing of the information in short-term plasticity model is not affected by it.

\section{Introduction}

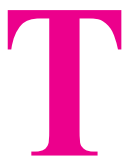

he Basolateral Amygdala (BLA) acts as a critical region that can modulate learning and memory in the other brain regions, such as the hippocampus (McGaugh, 2004). BLA presents an activity-dependent effect on the synaptic efficacies within the hippocampal subregions' neural circuits (Eichenbaum, 2000). Some cells, like the granule cells, are excitatory in the dentate gyrus, while the others are inhibitory neural cells (Amaral, Scharfman, \& Lavenex, 2007). The pairedpulse patterns with different Inter-Pulse Intervals (IPI) technique has been widely used to assess the activities of the cellular population of local circuits in the hippocampal subregions, such as CA1 and the dentate gyrus (Seress, Frotscher, \& Ribak, 1989). It has been well demonstrated that the dentate granule cell excitability can be modulated by different extra hippocampal inputs synapsing principally on inhibitory interneurons in the dentate gyrus (Freund \& Buzsáki, 1996).

Furthermore, multiple brain studies have demonstrated that the basolateral amygdala plays a crucial role in the synaptic efficacies in the hippocampal formation (Ardeshiri, Hosseinmardi, \& Akbari, 2018; Li \& Richter-Levin, 2013). Furthermore, the local anesthetic tetracaine injection into the BLA attenuates the synaptic plasticity in the dentate gyrus synapses (Abe, Fujimoto, Akaishi, \& Misawa, 2009). Various neurotransmitter systems in the BLA seem to be involved in this modulatory process. Orexins are novel neuropeptides that have been proposed concerning learning and memory. Some limited neurons in the posterior lateral hypothalamic area secrete orexin peptides by the prepro-orexin polypeptide. There are two types of orexin peptides named orexin-A and orexin-B. Both peptides bind to different kinds of receptors belonging to the G-protein-coupled receptors family. Orexin-A can bind to the Orexin-1 (OX1R) or Orexin-2 Receptors (OX2R), while orexin-B can only bind to the OX2R (Sakurai et al., 1998).

Moreover, one of the primary and specific targets for the orexinergic system is the amygdala (Schmitt et al., 2012). Furthermore, the level of OX1R and OX2R mRNA expression is high in different amygdala nuclei (Sakurai, 2005). Various studies indicated the engagement of the OX1Rs in the reward circuits, while OX2Rs are reported to be engaged in the arousal/stress neural system (Mahler, Smith, Moorman, Sartor, \& Aston-Jones, 2012). The Orexinergic system has recognized roles in the sleep/wakefulness cycle, feeding behaviors, drug dependence, memory, and anxiety axis (Sakurai, 2007). Orexin inputs reach various brain structures, such as the thalamus, septal area, raphe nucleus, ventral tegmental area, and amygdala (Peyron et al., 1998; Richardson \& Aston-Jones, 2012). The inactivation of the orexin receptors of the BLA indicated a critical role that can affect long period synaptic plasticity in the synaptic spaces between the granular cells of the dentate gyrus (Ardeshiri et al., 2018) and passive avoidance memory (Ardeshiri, Hosseinmardi, \& Akbari, 2017). Our previously reported experimental data have emphasized the roles of the BLA orexin receptors using Long-Term Potentiation (LTP) analysis and long-term potentiation. However, the function of these receptors in the hippocampal local interneuron circuits and the short-term plasticity remains undiscovered. Therefore, for clarifying the underlying mechanisms, we assessed the effects of orexin receptors antagonism of the basolateral part of the amygdala on the interactions among the granular layer's interneurons. We used SB334867-A (SB) and TCS-OX2-29 (TCS) 
in these experiments. We applied paired-pulse patterns to the medial perforant pathway using 20 and 80 InterPulse Intervals (IPI) to clarify how these local changes are mediated. At the short inter-pulse interval, the population spike recorded from the principal cells in the dentate gyrus is depressed via a mechanism dependent on the Gamma-Aminobutyric Acid type A receptors (GABA-A receptors). The longer inter-pulse interval results in paired-pulse disinhibition (facilitation), i.e., caused by different mechanisms such as reducing the GABAergic inhibition through the presynaptic GABA-B receptors (Jedlicka, Deller, \& Schwarzacher, 2010).

\section{Methods}

\section{Animals}

Our subjects were adult albino male Wistar rats weighing 250 to 300 grams. Three animals were kept in each cage at the animal center of the Mazandaran University of medical sciences, with a 12:12 h light/dark cycle. The experiments and the tests were accomplished within the light period of the day-night circadian rhythm. The animal institute was equipped with automatic instruments which controlled the temperature $\left(25 \pm 2{ }^{\circ} \mathrm{C}\right)$ and humidity $(30 \%-70 \%)$. Food and water were freely accessible ad libitum. Ethical principles governing the conduct of experiments with animals were also reviewed and followed. Therefore, the current study was designed according to our university's instructions and ethical guidelines and the international approved guidelines for using laboratory animals. This investigation includes 6 groups with 6 rats in each.

\section{Method of animals' surgery}

After anesthetizing the animals by the urethane $(1.5 \mathrm{~g} /$ $\mathrm{kg}$, intraperitoneal), they were fixed in a stereotaxic instrument (Stoelting, USA). Two guide cannulas for administrating the treatment interventions were stabilized inside the BLA areas. The dental cement was used for fixing. The coordination of the BLA was chosen using the Paxinos and Watson atlas (anterior-posterior [AP]: $-2.7 \mathrm{~mm}$ from bregma; medial-lateral [ML]: $\pm 4.8 \mathrm{~mm}$ from midline; and dorsal-ventral [DV]: $7.7 \mathrm{~mm}$ from the surface of the skull) (Paxinos \& Watson, 2006).

For evoked potential recording from the hippocampus region, two bipolar stainless-steel, Teflon-coated electrodes (127 $\mu \mathrm{m}$ bare size, Advent Co., UK) were inserted in the right hemisphere of the rat brain. One was the stimulating electrode that was inserted in the right medial perforant pathway with the following coordination,
AP: 8.1, ML: 4.3, and DV: $3.5 \mathrm{~mm}$, and the other electrode was inserted inside the ipsilateral granular layer of the hippocampal formation, which is coordinated as AP: 3.8, ML: 2.4, and DV: 3-3.5 mm. The electrodes were placed and remained in the desired positions by gentle downward movements. The electrodes remained at the desired places for about 30 minutes to one hour before starting any stimulation.

\section{Treatments protocols}

Dimethyl Sulfoxide (DMSO) was used as the solvent for antagonists. Drugs or vehicles $(0.5 \mu \mathrm{L})$ were injected via guide cannulas (21 gauge) positioned at the bilateral BLA. For injecting the antagonists or DMSO, a $10-\mu \mathrm{L}$ Hamilton micro-syringe connected to the 27 -gauge injection needle through the polyethylene tube was used. Treatments were applied over a 2-min time, and then the needle has remained at least one additional minute in the position. In this way, the treatments did not withdraw through the canula. At the designed time, $12 \mu \mathrm{g} / 0.5 \mu \mathrm{L}$ of SB or $10 \mu \mathrm{g} / 0.5$ $\mu \mathrm{L}$ of TCS were infused bilaterally into the BLA. All the protocols of administrations and the doses were selected regarding the former investigations in the field (Ardeshiri et al., 2017). DMSO as the vehicle was administrated to the control group in this study. Based on previous literature, DMSO presented no impact on learning or synaptic plasticity when applied in the brain (Akbari, Motamedi, Davoodi, Noorbakhshnia, \& Ghanbarian, 2011; Bianchin, Mello e Souza, Medina, \& Izquierdo, 1999; Naghdi \& Asadollahi, 2004; Suzuki \& Okada, 2010).

\section{Recording of field potentials and paired-pulse}

The rats were fixed in a stereotaxic device inside the Faraday cage to field potential recordings. Then, as mentioned above, two bipolar electrodes were fixed into the right perforant path and ipsilateral DG. Afterward, the evoked potential of many neurons of DG was recorded. For this aim, the Test Stimulus (TS) was delivered to the medial perforant pathway with an interval of 10 seconds. For each rat, the adequate intensity of the stimulus was plotted according to the Input/Output (I/O) profile for that rat. Stimulus intensities between 100 and $1000 \mu \mathrm{A}$ were applied to the perforant pathway.

Furthermore, the evoked potentials were plotted as an I/O profile. The measurable value in this protocol was the minimum stimulus intensity with the maximum Population Spike (PS) amplitude. Moreover, $40 \%$ of this intensity was suitable for recording the basal activity and paired responses (Nategh, Nikseresht, Khodagholi, \& Motamedi, 2016). 
The paired-pulse depression/facilitation was recorded using 10 repetitive stimuli at 20 , and $80 \mathrm{~ms}$ inter-pulse intervals were applied to the projection fibers to the granular layer. Then, the Paired-pulse Index (PPI), as the main parameter of the short-term plasticity, was calculated. The applied values are expressed in percentage $(\mathrm{PPI} \%=\mathrm{PS} 2 \times 100 /$ PS1) (Austin, Bronzino, \& Morgane, 1989).

The stimuli were sent by a homemade device named D3111 Science Beam to the projection fibers to the granular layer. The evoked potentials from the DG layer neurons were amplified and recorded by the same device.

\section{Experimental design}

Initially, the basal function of the granular cells was assessed. After stable recording, paired-pulse stimulations at $20 \mathrm{~ms}$ and $80 \mathrm{~ms}$ inter-pulse intervals were applied, and the responses were recorded. To determine the effect of orexin receptors antagonism of the BLA region on short-term plasticity, the SB, TCS (OX1R and OX2R antagonists, respectively), or DMSO were delivered through the cannulas. Finally, $15 \mathrm{~min}$ after the delivery of drugs or vehicles, the PPI responses were re-recorded with the same IPI.

\section{Verifying the injection}

To define the cannulas' place, $0.5 \mu \mathrm{L}$ methylene blue was injected through the cannulas. For ethic, the animals were deeply anesthetized and then sacrificed. The brains were extracted and kept in paraformaldehyde for 3-4 days until brain sectioning. A vibro-slice device (Campden, USA) was used to prepare $100-\mu \mathrm{m}$ sections of the brain. The sites of the injections were matched regarding the Paxinos and Watson. If the cannula was not in the right place, the data of that animal had been excluded.

The repeated-measured Analysis of Variance (ANOVA) compared the paired-pulse index at different interpulse intervals. Sidak's multiple comparisons test was used as further analysis. The extracted results were displayed as the Mean \pm SEM. Furthermore, $\mathrm{P}<0.05$ was considered significant.

\section{Results}

Figure 1-A shows the granular layer with the sign of the recording electrode. One side of the brain section, i.e., highlighted by the injection of methylene blue before decapitation, is demonstrated in Figure 1-B. The distinguished areas present the location of the BLA (Figure 1-C).
For all animals in all the groups, the I/O profile was plotted before stabilizing the synaptic responses and before any treatment was received (Figure 2).

The results of DMSO or SB administration into the BLA area on paired-pulse responses with 20 and $80 \mathrm{~ms}$ interpulse intervals in DG

After stabilizing the synaptic response and determining the I/O curve, the SB or DMSO was injected into the BLA region. After $15 \mathrm{~min}$, paired-pulse stimuli were applied with 20 and $80 \mathrm{~ms}$ intervals, and synaptic responses were recorded from the DG. Next, the paired response index (PPI $\%$ ) was measured based on the PS range. The ANOVA data indicated that SB had no significant effect on $\mathrm{PPI} \%$ index at $20 \mathrm{~ms}\left(\mathrm{~F}_{1,20}=1.3 ; \mathrm{P}=0.91\right)$ or $80 \mathrm{~ms}\left(\mathrm{~F}_{1,20}=1.3 ; \mathrm{P}=0.39\right)$ compared to the DMSO group (Figure $3-\mathrm{B}$ ). After the administration of DMSO or SB, the traces of Paired-pulse (PP) responses were recorded (Figure 3-C). In the OX1R antagonist treated group, the PP recording did not significantly affect 20 and $80 \mathrm{~ms}$ stimuli (SB-334867-A) relative to the control group ( $\mathrm{n}=6$ /group).

The results of DMSO or TCS administration into the BLA area, at 20 and $80-\mathrm{ms}$ inter-pulse intervals

The paired-pulse index at $20\left(\mathrm{~F}_{1,20}=1.8 ; \mathrm{P}=0.56\right)$ and 80 ms IPI $\left(\mathrm{F}_{1,20}=1.8 ; \mathrm{P}=0.57\right)$ before and after the microinjection of the OX2R antagonist (TCS-OX2-29) showed no statistically significant differences in comparison with DMSO treated group (Figure 4-B). After administrating DMSO or TCS, the traces of PP were recorded (Figure 4-C).Data analysis demonstrated no significant differences in the OX2R antagonist treated animals (TCSOX2-29) at 20 and 80 milliseconds relative to the DMSO treated animals ( $\mathrm{n}=6$ /group).

\section{Discussion}

Injecting orexin-1 and 2 receptors antagonists, i.e., SB334867-A or TCS-OX2-29, into the BLA nucleus could not affect short-term synaptic efficacy in the hippocampal subregions (DG) measured by the PP protocols using 20 and $80 \mathrm{~ms}$ inter-pulse interval stimulations.

The transmission of neural mediators through DG can be partly controlled by changing the internal activities of dentate gyrus local circuits to create the prerequisites for a particular response. The neuronal synapse of the perforant path-granular cell in the dentate gyrus displays a facilitator or inhibitory response given a time interval (Alger \& Teyler, 1976; Austin et al., 1989). 


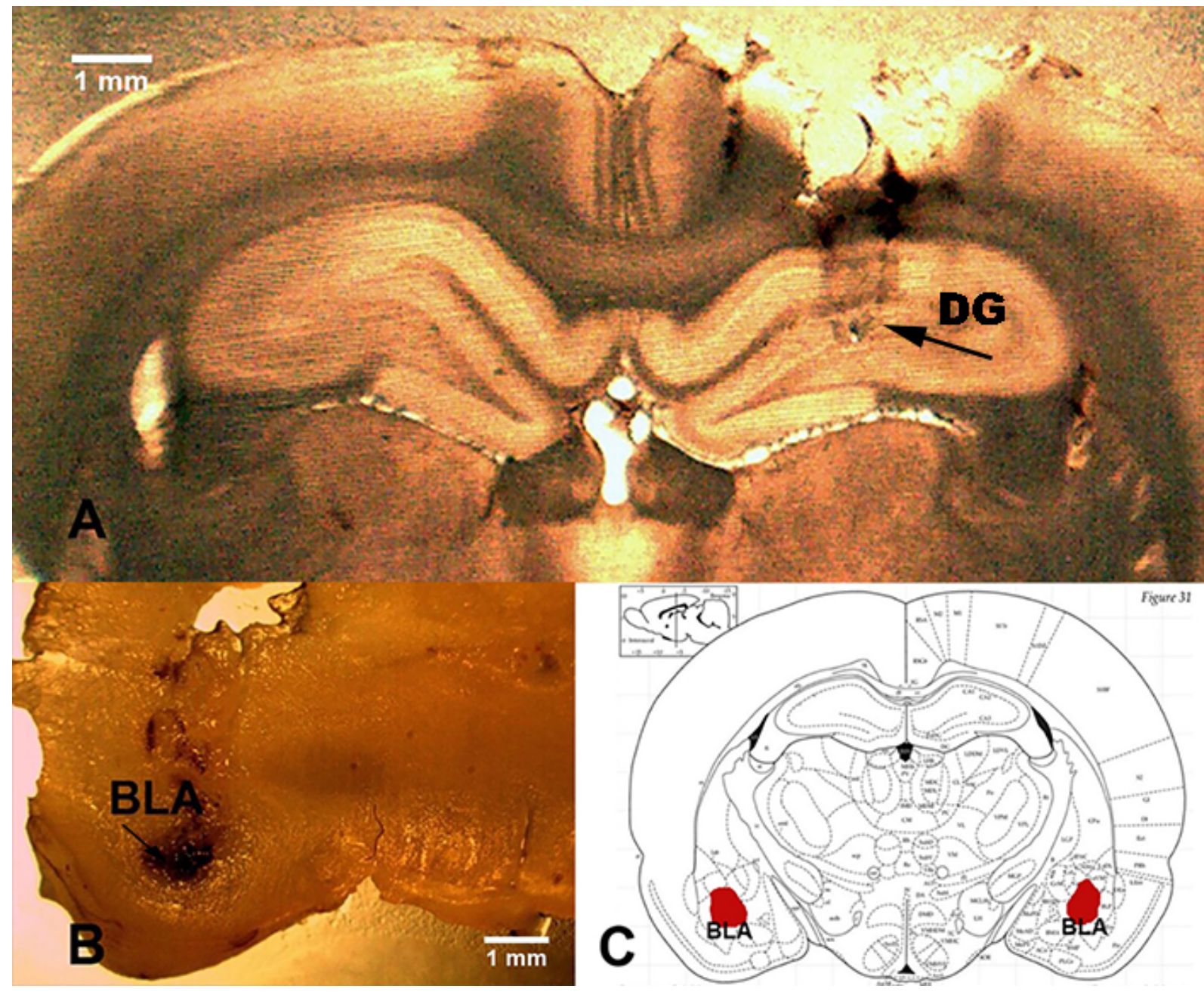

NEUR:SCIENCE

Figure 1. The sign of the recording electrode in DG granule cell layer (A), the site of the injection into the BLA area (B), and the site of antagonist injections into the BLA (C)

In this study, the paired-pulse index in the anesthetized rat in SB treated group at $20 \mathrm{~ms}$ IPI could completely inhibit the second population spike that was not different from the DMSO group. These results might have been generated for several reasons. It seems that the entorhino-dentate projections originate from layers IVVI likely terminate on GABAergic inhibitory neurons (Deller, Martinez, Nitsch, \& Frotscher, 1996). These fibers are glutamatergic (White, Nadler, Hamberger, Cotman, \& Cummins, 1977) and probably do not contain GABA neurotransmitters (Deller et al., 1996). Thus, the entorhino-dentate projection fibers with a feed-forward mechanism might excite the inhibitory cells with a lower threshold than the principal cells. These cells, in turn, may induce Inhibitory Postsynaptic Potentials (IPSPs) and suppress the spontaneous firing of the principal cells (Buzsáki, 1984). The orexin-1 receptors antagonism of the BLA could probably impair the function of the neu- rotransmitters and their subsequent activities; however, these impairments could not prevent the activity of the inhibitory cells of the granular layer named the basket cells were excited by the paired-pulse stimulation.

Furthermore, the excitability of granule cells might be controlled by the tonic discharge of another inhibitory network outside the dentate gyrus (Szabo et al., 2017). For instance, Freund et al. reported that both the cholinergic and GABAergic neurons were projected from the medial septum to the hippocampus, which extends finally to the dentate gyrus. According to their results, GABAergic fibers formed the synapse directly on GABAergic neurons in the dentate gyrus (Freund \& Antal, 1988). Therefore, projection of these GABAergic pathways from the medial septum probably was active during orexin receptor antagonism of the BLA and acted on inhibitory inter-neuronal activity in the dentate gyrus 


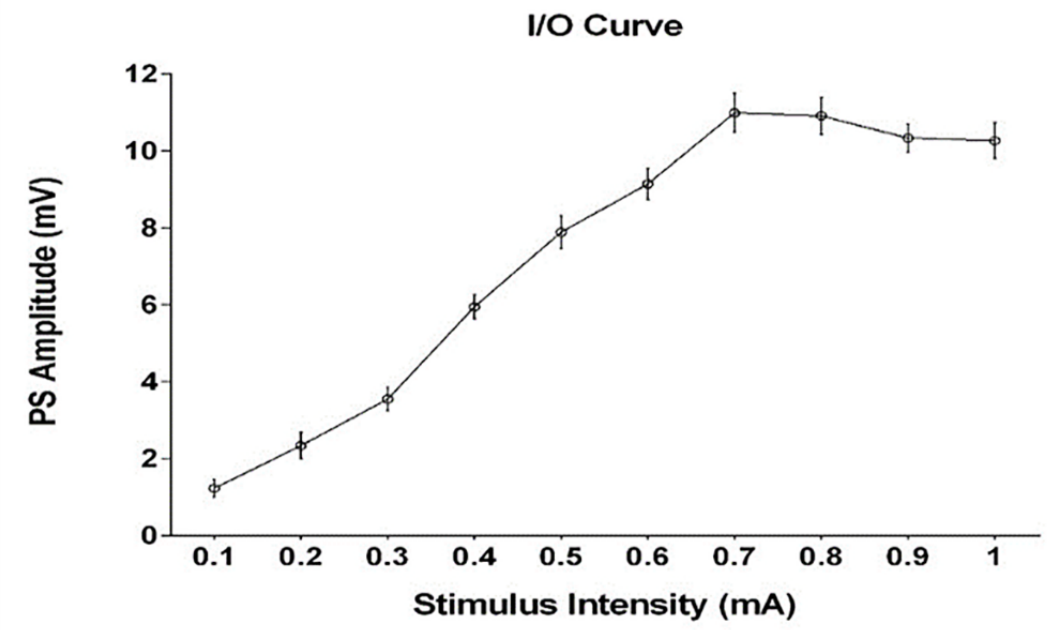

Figure 2. The I/O profile (intensity/voltage) at different stimulus intensities in the anesthetized animals ( $\mathrm{n}=36$ )

NEUR SCIENCE

during the paired response recordings. Besides, it has been shown that the amplitudes of population spikes in SB-injected rats gradually recovered toward the baseline recorded response level after $20 \mathrm{~min}$ (Ardeshiri et al., 2018). Investigating the paired-pulse responses more than 15 min after an antagonist injection might have enabled used to observe more effects.

As a measure of inhibitory/facilitatory modulation in DG local circuits taken from adult anesthetized rats before and after TCS (OX2R antagonist) infusion into the
BLA, the PPI showed a similar characteristic dependent on IPI, compared to the DMSO. The absence of any effect on the inhibitory/facilitatory modulation after TCS administration rules out the possibility that local synaptic transmission was affected by orexin-2 receptors of the BLA. These results demonstrate that manipulating the orexin receptors of the BLA could not affect the paired-pulse activities in the granular layer. Our former investigations also confirmed that the impact of the orexinergic projection fibers of the BLA on the long-term
(A)

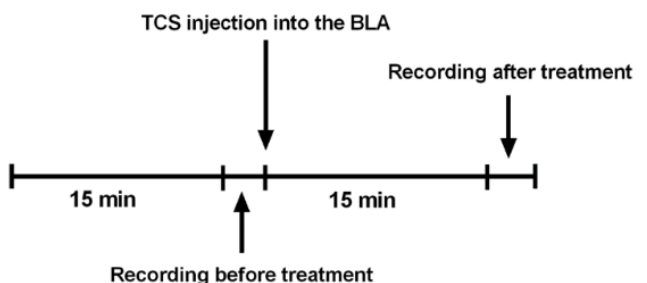

(B)

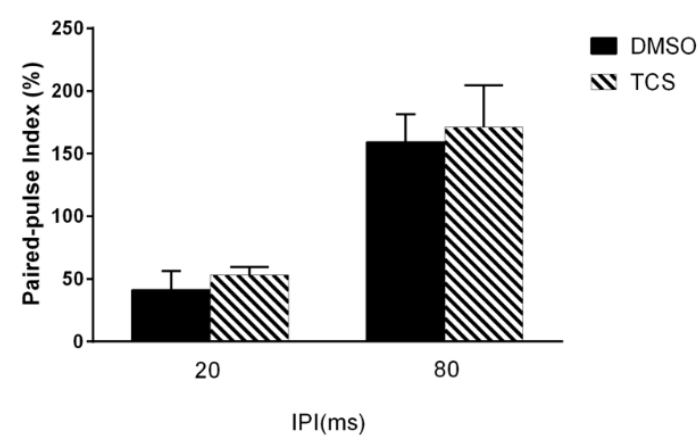

(C)

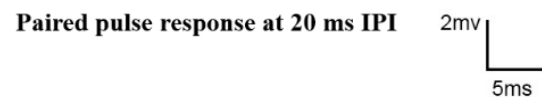

DMSO

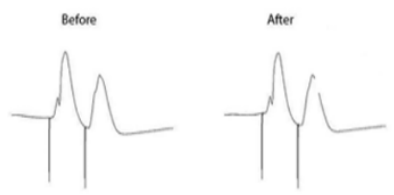

TCS

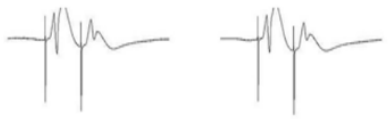

Paired pulse response at $80 \mathrm{~ms}$ IPI

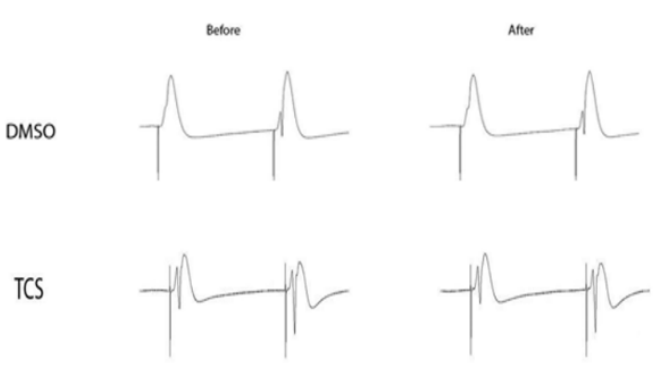

NEUR SCIENCE

Figure 4. The timeline of study (A), paired-pulse plasticity was measured by 20 and $80 \mathrm{~ms}$ inter-pulse intervals in DG (B), real traces of the paired responses were recorded during DMSO or TCS (C) administration 
(A)
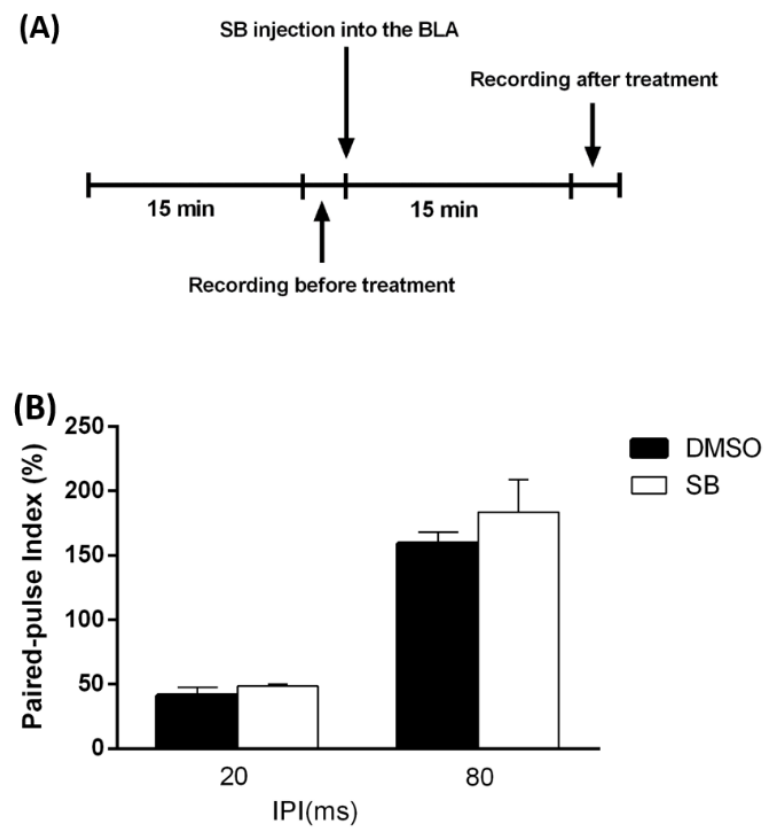

(C)

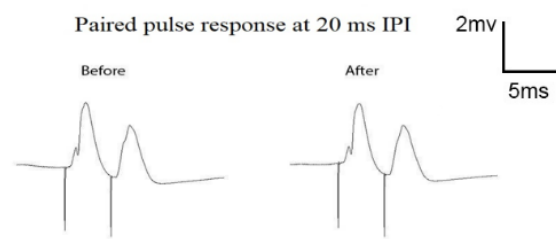

SB

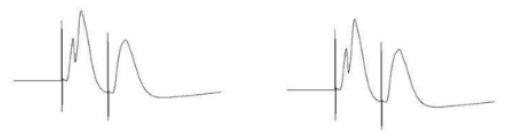

Paired pulse response at $80 \mathrm{~ms}$ IP

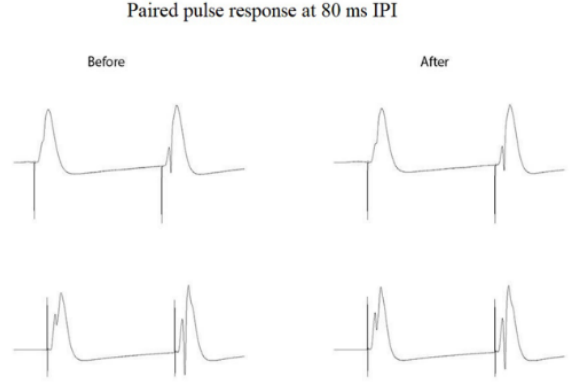

NEUR SCIENCE

Figure 3. The timeline of study (A), short-term plasticity measured the following 20 and $80 \mathrm{~ms}$ inter-pulse intervals in DG (B), real traces of the paired-pulse responses before and after the administration of the treatments $(C)$

activity (Ardeshiri et al., 2018) happened independent of the short-term plasticity.

The results probably support the idea that to make separate memory traces, separate neuronal systems with different neurotransmitters are activated during the parallel processing of various forms of memory (Bianchin et al., 1999). In addition, the unchanged hippocampal local circuit activity observed in this study following orexin receptors antagonism of BLA was not necessary for the LTP induction previously reported by us, and LTP induction was not made secondarily to this alteration (Ardeshiri et al., 2018). An emotional event probably triggers an orexinergic system of the BLA and then affects long-term memory. However, the local circuits act to their normal state to create an adequate balance between inhibitor and facilitator state to form an appropriate behavior.

\section{Conclusion}

Due to not observing any significant changes in the short form of synaptic plasticity, after inactivation of the orexin system of the BLA, we hypothesize that the orexinergic fibers to the basolateral part of the amygdala influence the long-term synaptic efficacy, but the primary processing of information in short-term plasticity model is not affected by the same system. The elementary pro- cessing of the information by the amygdala might happen through the action of other neurotransmitter systems.

\section{Ethical Considerations}

\section{Compliance with ethical guidelines}

The study was approved by the ethics committee of Shahid Beheshti University of Medical Sciences, Tehran (Code: IR.SBMU.SM.REC.1394.64).

Funding

The study was suported by a grant at Mazandaran University of Medical Sciences (No. 2339), and Shaheed Beheshti University of Medical Sciences (No. 564).

Authors' contributions

All authors equally contributed to preparing this article.

\section{Conflict of interest}

The authors declared no conflict of interest. 


\section{Refrences}

Abe, K., Fujimoto, T., Akaishi, T., \& Misawa, M. (2009). Basolateral amygdala D1-and D2-dopaminergic system promotes the formation of long-term potentiation in the dentate gyrus of anesthetized rats. Progress in Neuro-Psychopharmacology and Biological Psychiatry, 33(3), 552-6. [DOI:10.1016/j.pnpbp.2009.02.011] [PMID]

Akbari, E., Motamedi, F., Davoodi, F. G., Noorbakhshnia, M., \& Ghanbarian, E. (2011). Orexin-1 receptor mediates longterm potentiation in the dentate gyrus area of freely moving rats. Behavioural Brain Research, 216(1), 375-80. [DOI:10.1016/j. bbr.2010.08.017] [PMID]

Alger, B.E., \& Teyler, T. J. (1976). Long-term and shortterm plasticity in the CA1, CA3, and dentate regions of the rat hippocampal slice. Brain Research, 110(3), 463-80. [DOI:10.1016/0006-8993(76)90858-1] [PMID]

Amaral, D. G., Scharfman, H. E., \& Lavenex, P. (2007). The dentate gyrus: Fundamental neuroanatomical organization (dentate gyrus for dummies). Progress in Brain Research, 163, 3-790. [DOI:10.1016/S0079-6123(07)63001-5] [PMID] [PMCID]

Ardeshiri, M.R., Hosseinmardi, N., \& Akbari, E. (2017). The effect of orexin 1 and orexin 2 receptors antagonisms in the basolateral amygdala on memory processing in a passive avoidance task. Physiology \& Behavior, 174, 42-8. [DOI:10.1016/j.physbeh.2017.03.004] [PMID]

Ardeshiri, M. R., Hosseinmardi, N., \& Akbari, E. (2018). Orexin 1 and orexin 2 receptor antagonism in the basolateral amygdala modulate long-term potentiation of the population spike in the perforant path-dentate gyrus-evoked field potential in rats. Neurobiology of Learning and Memory, 149, 98-106. [DOI:10.1016/j.nlm.2018.02.024] [PMID]

Austin, K. B., Bronzino, J., \& Morgane, P. J. (1989). Paired-pulse facilitation and inhibition in the dentate gyrus is dependent on behavioral state. Experimental Brain Research, 77(3), 594-604. [DOI:10.1007/BF00249612] [PMID]

Bianchin, M., Mello e Souza, T., Medina, J. H., \& Izquierdo, I. (1999). The amygdala is involved in the modulation of longterm memory, but not in working or short-term memory. Neurobiology of Learning and Memory, 71(2), 127-31. [DOI:10.1006/ nlme.1998.3881] [PMID]

Buzsáki, G. (1984). Feed-forward inhibition in the hippocampal formation. Progress in Neurobiology, 22(2), 131-53. [DOI:10.1016/0301-0082(84)90023-6] [PMID]

10. Deller, T., Martinez, A., Nitsch, R., \& Frotscher, M. (1996) A novel entorhinal projection to the rat dentate gyrus: Direct innervation of proximal dendrites and cell bodies of granule cells and GABAergic neurons. Journal of Neuroscience, 16(10), 3322-33. [DOI:10.1523/JNEUROSCI.16-10-03322.1996] [PMID] [PMCID]

Eichenbaum, H. (2000). A cortical-hippocampal system for declarative memory. Nature Reviews Neuroscience, 1(1), 41-50. [DOI:10.1038/35036213] [PMID]

Freund, T. F., \& Antal, M. (1988). GABA-containing neurons in the septum control inhibitory interneurons in the hippocampus. Nature, 336(6195), 170-3. [DOI:10.1038/336170a0] [PMID]

Freund, T. F., \& Buzsáki, G. (1996). Interneurons of the hippocampus. Hippocampus, 6(4), 347-470. [DOI:10.1002/ (SICI)1098-1063(1996)6:43.0.CO;2-I] [PMID]
Jedlicka, P., Deller, T., \& Schwarzacher, S. W. (2010). Computational modeling of GABA A receptor-mediated paired-pulse inhibition in the dentate gyrus. Journal of Computational Neuroscience, 29(3) 509-19. [DOI:10.1007/s10827-010-0214-y] [PMID]

Li, Z., \& Richter-Levin, G. (2013). Priming stimulation of basal but not lateral amygdala affects long-term potentiation in the rat dentate gyrus in vivo. Neuroscience, 246, 13-21. [DOI:10.1016/j. neuroscience.2013.03.059] [PMID]

Mahler, S. V., Smith, R. J., Moorman, D. E., Sartor, G. C., \& AstonJones, G. (2012). Multiple roles for orexin/hypocretin in addiction. In Progress in Brain Research, 198, 79-121 [DOI:10.1016/B9780-444-59489-1.00007-0] [PMID] [PMCID]

McGaugh, J. L. (2004). The amygdala modulates the consolidation of memories of emotionally arousing experiences. Annual Review of Neuroscience, 27, 1-28. [DOI:10.1146/annurev.neuro.27.070203.144157] [PMID]

Naghdi, N., \& Asadollahi, A. (2004). Genomic and nongenomic effects of intrahippocampal microinjection of testosterone on long-term memory in male adult rats. Behavioural Brain Research, 153(1), 1-6. [DOI:10.1016/j.bbr.2003.10.027] [PMID]

Nategh, M., Nikseresht, S., Khodagholi, F., \& Motamedi, F. (2016) Inactivation of nucleus incertus impairs passive avoidance learning and long term potentiation of the population spike in the perforant path-dentate gyrus evoked field potentials in rats. Neurobiology of Learning and Memory, 130, 185-93. [DOI:10.1016/]. nlm.2016.02.012] [PMID]

Paxinos, G., \& Watson, C. (2006). The rat brain in stereotaxic coordinates. Amsterdam: Elsevier Science. https://www.google.com/ books/edition/The_Rat_Brain_in_Stereotaxic_Coor

Peyron, C., Tighe, D. K., Van Den Pol, A. N., De Lecea, L., Heller H. C., \& Sutcliffe, J. G., et al. (1998). Neurons containing hypocretin (orexin) project to multiple neuronal systems. Journal of Neuroscience, 18(23), 9996-10015. [DOI:10.1523/JNEUROSCI.18-23-09996.1998] [PMID] [PMCID]

Richardson, K. A., \& Aston-Jones, G. (2012). Lateral hypothalamic orexin/hypocretin neurons that project to ventral tegmental area are differentially activated with morphine preference. Journal of Neuroscience, 32(11), 3809-17. [DOI:10.1523/JNEUROSCI.3917-11.2012] [PMID] [PMCID]

Sakurai, T. (2005) Orexin and Orexin Receptors. In L. de Lecea, \& J. G. Sutcliffe (Eds.), Hypocretins (pp. 13-23). Boston: Springer. [DOI:10.1007/0-387-25446-3_2]

Sakurai, T. (2007). The neural circuit of orexin (hypocretin): Main taining sleep and wakefulness. Nature Reviews Neuroscience, 8(3), 171-81. [DOI:10.1038/nrn2092] [PMID]

Sakurai, T., Amemiya, A., Ishii, M., Matsuzaki, I., Chemelli, R. M., \& Tanaka, H., et al. (1998). Orexins and orexin receptors: A family of hypothalamic neuropeptides and $\mathrm{G}$ protein-coupled receptors that regulate feeding behavior. Cell, 92(4), 573-85. [DOI:10.1016/ S0092-8674(00)80949-6] [PMID]

Schmitt, O., Usunoff, K. G., Lazarov, N. E., Itzev, D. E., Eipert, P., \& Rolfs, A., et al. (2012). Orexinergic innervation of the extended amygdala and basal ganglia in the rat. Brain Structure and Function, 217(2), 233-56. [DOI:10.1007/s00429-011-0343-8] [PMID]

Seress, L., Frotscher, M., \& Ribak, C. E. (1989). Local circuit neurons in both the dentate gyrus and Ammon's horn establish synaptic connections with principal neurons in five day old rats: A morphological basis for inhibition in early development. Experimental Brain Research, 78(1), 1-9. [DOI:10.1007/BF00230680] [PMID] 
Suzuki, E., \& Okada, T. (2010). Group I metabotropic glutamate receptors are involved in TEA-induced long-term potentiation at mossy fiber-CA3 synapses in the rat hippocampus. Brain Research, 1313, 45-52. [DOI:10.1016/j.brainres.2009.11.059] [PMID]

Szabo, G. G., Du, X., Oijala, M., Varga, C., Parent, J. M., \& Soltesz, I. (2017). Extended interneuronal network of the dentate gyrus. Cell Reports, 20(6), 1262-8. [DOI:10.1016/j.celrep.2017.07.042] [PMID] [PMCID]

White, W. F., Nadler, J. V., Hamberger, A., Cotman, C W., \& CUMMINS, J. T. (1977). Glutamate as transmitter of hippocampal perforant path. Nature, 270(5635), 356. [DOI:10.1038/270356a0] [PMID] 
This Page Intentionally Left Blank 